\title{
A New Alternating Segment Crank-Nicolson Scheme for the Fourth-Order Parabolic Equation
}

\author{
Ge-yang Guo ${ }^{1}$ and Bo Liu ${ }^{2}$ \\ ${ }^{1}$ Department of Mathematics, Tianjin University of Technology and Education, Tianjin 300222, China \\ ${ }^{2}$ College of Mathematics, Jilin University, Changchun 130012, China \\ Correspondence should be addressed to Ge-yang Guo; guogeyang2000@sina.com
}

Received 1 June 2013; Accepted 2 July 2013

Academic Editors: J. A. Ferreira and E. Yee

Copyright (c) 2013 G.-y. Guo and B. Liu. This is an open access article distributed under the Creative Commons Attribution License, which permits unrestricted use, distribution, and reproduction in any medium, provided the original work is properly cited.

\begin{abstract}
A group of asymmetric difference schemes to approach the fourth-order parabolic equation is given. According to these schemes and the Crank-Nicolson scheme, an alternating segment Crank-Nicolson scheme with intrinsic parallelism is constructed. The truncation errors and the stability are discussed. Numerical simulations show that this new scheme has unconditional stability and high accuracy and convergency, and it is in preference to the implicit scheme method.
\end{abstract}

\section{Introduction}

With the rapid development of high-performance computers, the need to construct parallel algorithms has long been desired. In recent years, the alternating schemes have been studied extensively in the literature. In 1983, Evans and Abdullah first developed the Alternating Group Explicit (AGE) scheme [1] for parabolic equation, which shows that it is possible to design parallel difference method by constructing a new difference scheme. Afterward, using the explicit scheme, the implicit scheme, and the Crank-Nicolson scheme, the Alternating Segment Explicit-Implicit (ASE-I) scheme [2] and the Alternating Segment Crank-Nicolson (ASC-N) scheme [3] were proposed. The results of numerical examples show that these schemes are unconditionally stable and have high accuracy. Currently, the alternating technology has been extended to dispersive equation [4-8], convectiondiffusion equation [9], Burgers equation [10], nonlinear three-order KdV equation [11] and fourth-order parabolic $[12,13]$.

The Kuramoto-Sivashinsky equation (K-S) $[14,15]$ is well-known as one of the mathematical equations which models the reaction-diffusion systems, flame propagation, and viscous flow problems. During recent years, many authors have focused on solving this equation numerically and analytically [16-18]. However, the parallel difference method for this equation has not been found. In this paper, we present the alternating segment Crank-Nicolson scheme for the following fourth-order parabolic equation:

$$
\frac{\partial u}{\partial t}+a \frac{\partial^{4} u}{\partial x^{4}}+\varepsilon \frac{\partial^{2} u}{\partial x^{2}}=0,
$$

which is the high order part of the linear K-S equation. We hope that the result of this paper makes an essential contribution in this direction.

We consider the following problem:

$$
\frac{\partial u}{\partial t}+a \frac{\partial^{4} u}{\partial x^{4}}+\varepsilon \frac{\partial^{2} u}{\partial x^{2}}=0, \quad x \in[0, L], t \in[0, T] .
$$

With the initial condition

$$
u(x, 0)=u_{0}(x), \quad x \in[0, L],
$$

and the boundary conditions

$$
u(0, t)=u_{x x}(0, t)=u(L, t)=u_{x x}(L, t)=0, \quad t \in[0, T],
$$

where $u_{0}(x)$ is a given function and $\varepsilon$ and $a$ are constants.

The plan of this paper is as follows. In Section 2, some basic schemes are given and the ASC-N scheme is developed. In Section 3, the error analysis and the stability analysis are discussed. In Section 4, numerical simulations are performed. Finally, a brief conclusion is given. 


\section{New Alternating Segment Crank-Nicolson Scheme}

2.1. Some Basic Schemes. Divide the domain of definition $[0, L] \times[0, T]$ by parallel lines $x=x_{j}=j h(j=0,1,2 \ldots, M)$, $t=t^{n}=n \tau(n=0,1,2 \ldots, N)$, where $h=L / M$ is the space mesh length and $\tau=T / N$ is the time mesh length. $M$ and $N$ are positive integers. We use $u_{j}^{n}$ to represent the approximate solution of $u\left(x_{j}, t^{n}\right)$, where $u(x, t)$ represents the exact solution of (2). We first introduce eight asymmetric schemes (5)-(12) (see the stencils in Figure 1) and CrankNicolson scheme (13) for (2):

$$
\begin{aligned}
& \frac{r}{2} u_{j+2}^{n+1}-\left(r+\frac{\bar{r}}{2}\right) u_{j+1}^{n+1}+\left(1+\frac{r}{2}+\frac{\bar{r}}{2}\right) u_{j}^{n+1} \\
& =-\frac{r}{2} u_{j+2}^{n}+\left(3 r+\frac{\bar{r}}{2}\right) u_{j+1}^{n}+\left(1-\frac{11 r}{2}-\frac{3 \bar{r}}{2}\right) u_{j}^{n} \\
& +(4 r+\bar{r}) u_{j-1}^{n}-r u_{j-2}^{n} \text {, } \\
& \left(1+\frac{r}{2}+\frac{\bar{r}}{2}\right) u_{j}^{n+1}-\left(r+\frac{\bar{r}}{2}\right) u_{j-1}^{n+1}+\frac{r}{2} u_{j-2}^{n+1} \\
& =-r u_{j+2}^{n}+(4 r+\bar{r}) u_{j+1}^{n}+\left(1-\frac{11 r}{2}-\frac{3 \bar{r}}{2}\right) u_{j}^{n} \\
& +\left(3 r+\frac{\bar{r}}{2}\right) u_{j-1}^{n}-\frac{r}{2} u_{j-2}^{n}, \\
& \frac{r}{2} u_{j+2}^{n+1}-\left(3 r+\frac{\bar{r}}{2}\right) u_{j+1}^{n+1}+\left(1+\frac{11 r}{2}+\frac{3 \bar{r}}{2}\right) u_{j}^{n+1} \\
& -(4 r+\bar{r}) u_{j-1}^{n+1}+r u_{j-2}^{n+1} \\
& =-\frac{r}{2} u_{j+2}^{n}+\left(r+\frac{\bar{r}}{2}\right) u_{j+1}^{n}+\left(1-\frac{r}{2}-\frac{\bar{r}}{2}\right) u_{j}^{n}, \\
& r u_{j+2}^{n+1}-(4 r+\bar{r}) u_{j+1}^{n+1}+\left(1+\frac{11 r}{2}+\frac{3 \bar{r}}{2}\right) u_{j}^{n+1} \\
& -\left(3 r+\frac{\bar{r}}{2}\right) u_{j-1}^{n+1}+\frac{r}{2} u_{j-2}^{n+1} \\
& =\left(1-\frac{r}{2}-\frac{\bar{r}}{2}\right) u_{j}^{n}+\left(r+\frac{\bar{r}}{2}\right) u_{j-1}^{n}-\frac{r}{2} u_{j-2}^{n}, \\
& \frac{r}{2} u_{j+2}^{n+1}-\left(2 r+\frac{\bar{r}}{2}\right) u_{j+1}^{n+1} \\
& +\left(1+\frac{5 r}{2}+\bar{r}\right) u_{j}^{n+1}-\left(r+\frac{\bar{r}}{2}\right) u_{j-1}^{n+1} \\
& =-\frac{r}{2} u_{j+2}^{n}+\left(2 r+\frac{\bar{r}}{2}\right) u_{j+1}^{n} \\
& +\left(1-\frac{7 r}{2}-\bar{r}\right) u_{j}^{n}+\left(3 r+\frac{\bar{r}}{2}\right) u_{j-1}^{n}-r u_{j-2}^{n}, \\
& -\left(r+\frac{\bar{r}}{2}\right) u_{j+1}^{n+1}+\left(1+\frac{5 r}{2}+\bar{r}\right) u_{j}^{n+1}
\end{aligned}
$$

$$
\begin{aligned}
& -\left(2 r+\frac{\bar{r}}{2}\right) u_{j-1}^{n+1}+\frac{r}{2} u_{j-2}^{n+1} \\
& =-r u_{j+2}^{n}+\left(3 r+\frac{\bar{r}}{2}\right) u_{j+1}^{n}+\left(1-\frac{7 r}{2}-\bar{r}\right) u_{j}^{n} \\
& +\left(2 r+\frac{\bar{r}}{2}\right) u_{j-1}^{n}-\frac{r}{2} u_{j-2}^{n}, \\
& \frac{r}{2} u_{j+2}^{n+1}-\left(2 r+\frac{\bar{r}}{2}\right) u_{j+1}^{n+1}+\left(1+\frac{7 r}{2}+\bar{r}\right) u_{j}^{n+1} \\
& -\left(3 r+\frac{\bar{r}}{2}\right) u_{j-1}^{n+1}+r u_{j-2}^{n+1} \\
& =-\frac{r}{2} u_{j+2}^{n}+\left(2 r+\frac{\bar{r}}{2}\right) u_{j+1}^{n} \\
& +\left(1-\frac{5 r}{2}-\bar{r}\right) u_{j}^{n}+\left(r+\frac{\bar{r}}{2}\right) u_{j-1}^{n}, \\
& r u_{j+2}^{n+1}-\left(3 r+\frac{\bar{r}}{2}\right) u_{j+1}^{n+1}+\left(1+\frac{7 r}{2}+\bar{r}\right) u_{j}^{n+1} \\
& -\left(2 r+\frac{\bar{r}}{2}\right) u_{j-1}^{n+1}+\frac{r}{2} u_{j-2}^{n+1} \\
& =\left(r+\frac{\bar{r}}{2}\right) u_{j+1}^{n}+\left(1-\frac{5 r}{2}-\bar{r}\right) u_{j}^{n} \\
& +\left(2 r+\frac{\bar{r}}{2}\right) u_{j-1}^{n}-\frac{r}{2} u_{j-2}^{n}, \\
& \frac{r}{2} u_{j+2}^{n+1}-\left(2 r+\frac{\bar{r}}{2}\right) u_{j+1}^{n+1}+(1+3 r+\bar{r}) u_{j}^{n+1} \\
& -\left(2 r+\frac{\bar{r}}{2}\right) u_{j-1}^{n+1}+\frac{r}{2} u_{j-2}^{n+1} \\
& =-\frac{r}{2} u_{j+2}^{n}+\left(2 r+\frac{\bar{r}}{2}\right) u_{j+1}^{n}+(1-3 r-\bar{r}) u_{j}^{n} \\
& +\left(2 r+\frac{\bar{r}}{2}\right) u_{j-1}^{n}-\frac{r}{2} u_{j-2}^{n} \text {, }
\end{aligned}
$$

where $r=a \tau / h^{4}, \bar{r}=\varepsilon \tau / h^{2}$.

\section{The ASC-N Scheme}

The parallel ASC-N scheme is constructed as follows. Assuming $M-1=2 \mathrm{Jl}$, we consider the model of the segments at the $(n+1)$ st and the $(n+2)$ nd time levels, where $n$ is an even number and $l>4$ is a positive integer. We divide the nodes of the $(n+1)$ st time level into $J$ independent computational segments; each segment in a left-to-right direction contains $2 l$ nodes, and the difference schemes of the $2 l$ nodes are arranged according to the

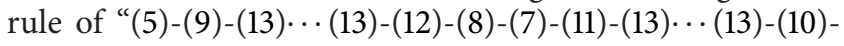
(6)". Similarly, we divide the nodes of the $(n+2)$ st time level into $J+1$ independent computational segments. The first segment contains $l$ nodes; the difference schemes of the $l$ nodes 

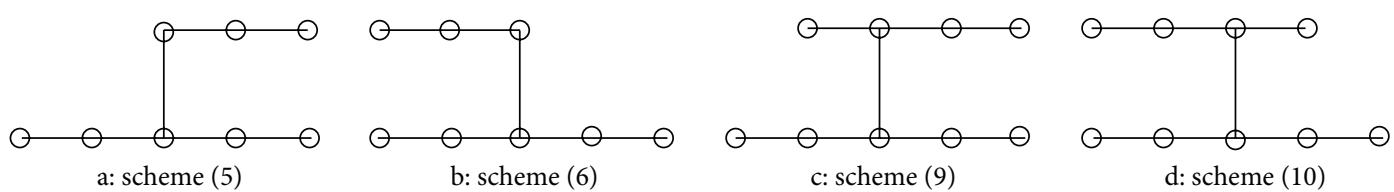

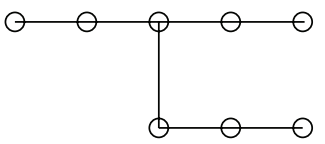

$a^{\prime}$ : scheme (7)
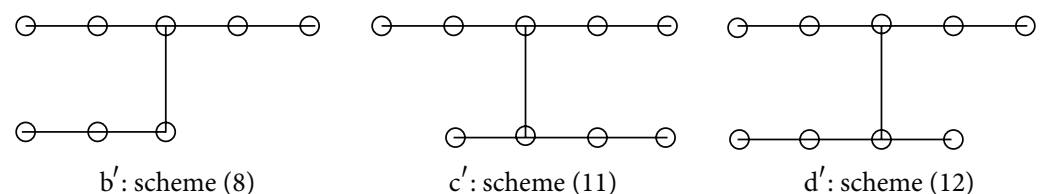

Figure 1: The diagram of asymmetric schemes (5)-(12).

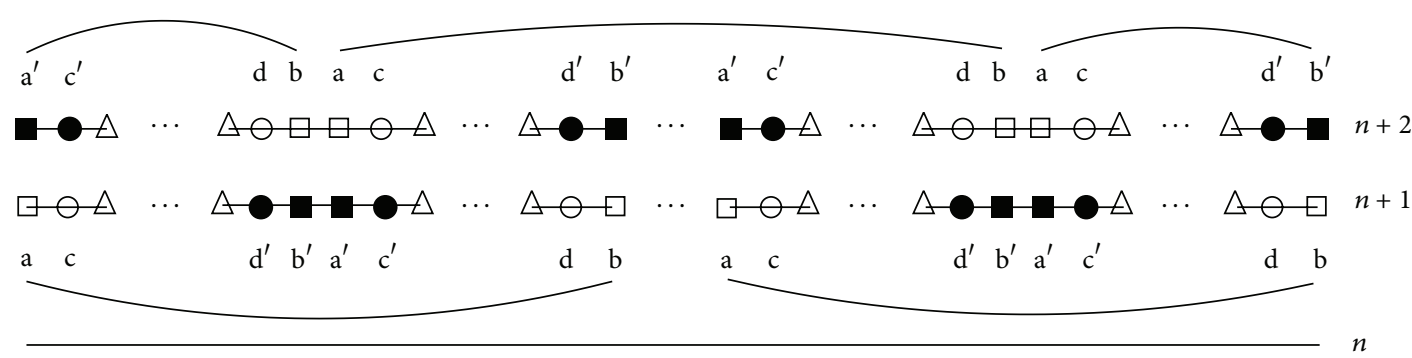

Figure 2: The diagram of the ASC-N scheme.

are arranged according to the rule of "(7)-(11)-(13) $\cdots(13)$ (10)-(6)". The $(J+1)$ st segment contains $l$ nodes; the difference schemes of the $l$ nodes are arranged according to the rule of "(5)-(9)-(13)‥(13)-(12)-(8)". The difference schemes of the segment of the $2 l$ nodes are arranged according to the

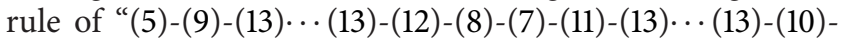
(6)". The asymmetric schemes are used alternately in pairs at two adjacent points of the $(n+1)$ st and the $(n+2)$ nd time levels. The rule is displayed in Figure 2, where $\square$ is used to denote the asymmetric scheme (5) or (6), $\mathbf{a}$ to denote the asymmetric scheme (7) or (8), 。 to denote the asymmetric scheme (9) or (10), - to denote the asymmetric scheme (11) or (12), and $\Delta$ to denote the Crank-Nicolson scheme. Hence, the matrix forms of the ASC-N scheme can be expressed as

$$
\begin{aligned}
& \left(\mathbf{I}+\mathbf{G}_{1}\right) \mathbf{U}^{n+1}=\left(\mathbf{I}-\mathbf{G}_{2}\right) \mathbf{U}^{n}, \quad n=0,2,4,6, \ldots, \\
& \left(\mathbf{I}+\mathbf{G}_{2}\right) \mathbf{U}^{n+2}=\left(\mathbf{I}-\mathbf{G}_{1}\right) \mathbf{U}^{n+1}, \quad n=0,2,4,6, \ldots,
\end{aligned}
$$

where $\mathbf{U}^{n}=\left(u_{1}^{n}, u_{2}^{n}, \ldots, u_{M-1}^{n}\right)^{T}, \mathbf{G}_{i}=r \mathbf{G}_{i}^{(1)}+\bar{r} \mathbf{G}_{i}^{(2)}, i=1,2$, and the matrices $\mathbf{G}_{i}^{(1)}$ and $\mathbf{G}_{i}^{(2)}$ are given by

$$
\begin{aligned}
& \mathbf{G}_{1}^{(1)}=\left(\begin{array}{lll}
\mathbf{Q}_{2 l} & & \\
& \ddots & \\
& & \mathbf{Q}_{2 l}
\end{array}\right), \\
& \mathbf{G}_{1}^{(2)}=\left(\begin{array}{lll}
\mathbf{P}_{2 l} & & \\
& \ddots & \\
& & \mathbf{P}_{2 l}
\end{array}\right), \\
& \mathbf{G}_{2}^{(1)}=\left(\begin{array}{ccccc}
\mathbf{Q}_{l}^{(l)} & & & & \overline{\mathbf{Q}}_{l}^{(r)} \\
& \mathbf{Q}_{2 l} & & & \\
& & \ddots & & \\
& & & \mathbf{Q}_{2 l} & \\
\overline{\mathbf{Q}}_{l}^{(l)} & & & & \mathbf{Q}_{l}^{(r)}
\end{array}\right) \text {, }
\end{aligned}
$$




$$
\begin{aligned}
& \mathbf{G}_{2}^{(2)}=\left(\begin{array}{ccccc}
\mathbf{P}_{l}^{(l)} & & & & \overline{\mathbf{P}}_{l}^{(r)} \\
& \mathbf{P}_{2 l} & & & \\
& & \ddots & & \\
\overline{\mathbf{P}}_{l}^{(l)} & & & & \mathbf{P}_{2 l} \\
\mathbf{P}_{l}^{(r)}
\end{array}\right)
\end{aligned}
$$

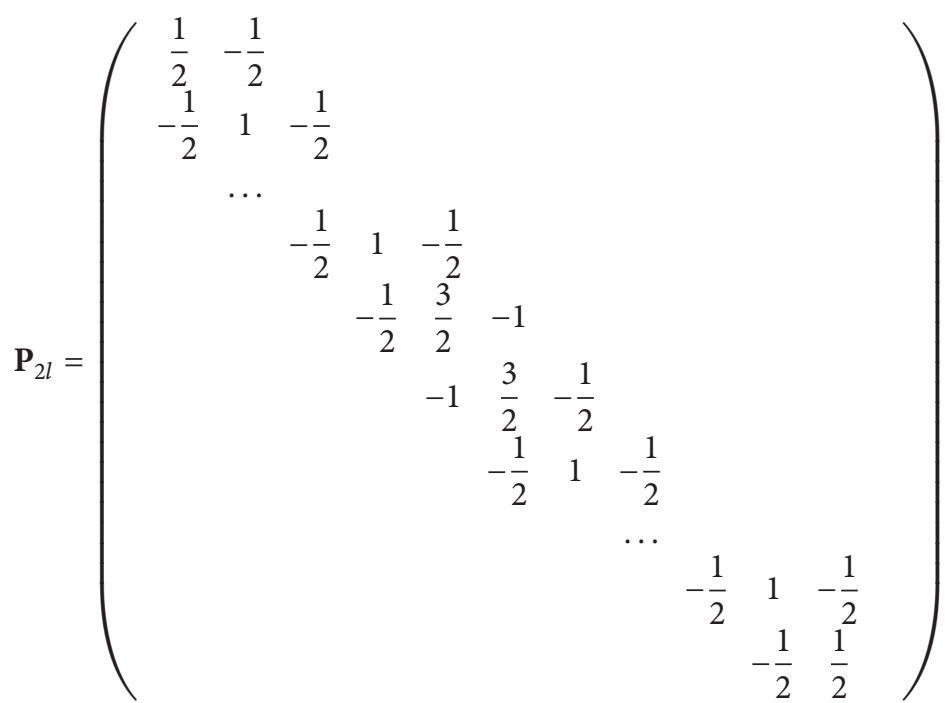

$$
\begin{aligned}
& =\left(\begin{array}{cc}
\mathbf{P}_{l}^{(r)} & \overline{\mathbf{P}}_{l}^{(l)} \\
\overline{\mathbf{P}}_{l}^{(r)} & \mathbf{P}_{l}^{(l)}
\end{array}\right),
\end{aligned}
$$

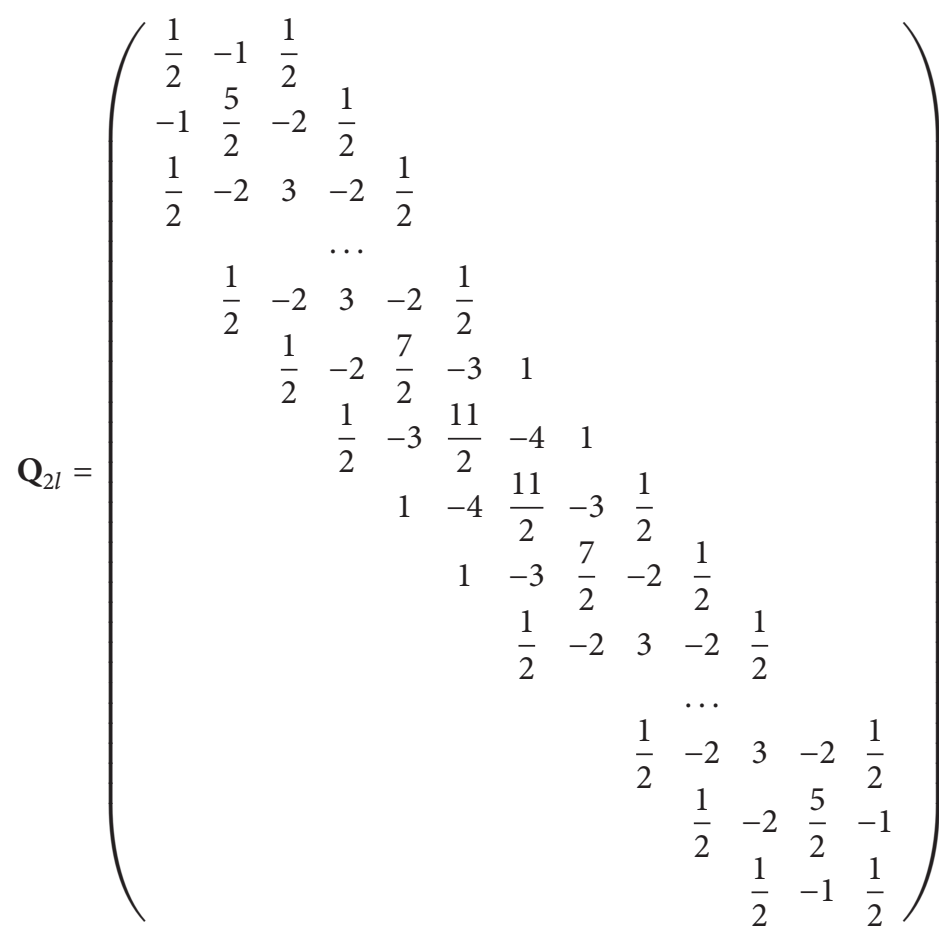

$$
\begin{aligned}
& =\left(\begin{array}{cc}
\mathbf{Q}_{l}^{(r)} & \overline{\mathbf{Q}}_{l}^{(l)} \\
\overline{\mathbf{Q}}_{l}^{(r)} & \mathbf{Q}_{l}^{(l)}
\end{array}\right) .
\end{aligned}
$$




\section{The Analysis of the Truncation Errors and Stability}

4.1. The Analysis of the Truncation Errors. In order to analyze the truncation errors, we change the scheme $a$ into the equivalent segment scheme of three levels; that is, adding scheme $a^{\prime}$ at the point $\left(x_{j}, t^{n+1}\right)$ and scheme $a$ at the point $\left(x_{j}, t^{n+2}\right)$ for two adjacent points of the $(n+1)$ st time level and $(n+2)$ nd time level, we obtain three-level scheme (17). Similarly, we obtain three-level schemes (18)-(24), respectively;

$$
\begin{aligned}
& \frac{r}{2} u_{j+2}^{n+2}-\left(r+\frac{\bar{r}}{2}\right) u_{j+1}^{n+2}+\left(1+\frac{r}{2}+\frac{\bar{r}}{2}\right) u_{j}^{n+2} \\
& =-\frac{r}{2} u_{j+2}^{n}+\left(r+\frac{\bar{r}}{2}\right) u_{j+1}^{n} \\
& +\left(1-\frac{r}{2}-\frac{\bar{r}}{2}\right) u_{j}^{n}-r u_{j+2}^{n+1} \\
& +(6 r+\bar{r}) u_{j+1}^{n+1}-(11 r+3 \bar{r}) u_{j}^{n+1} \\
& +(8 r+2 \bar{r}) u_{j-1}^{n+1}-2 r u_{j-2}^{n+1} \text {, } \\
& \left(1+\frac{r}{2}+\frac{\bar{r}}{2}\right) u_{j}^{n+2}-\left(r+\frac{\bar{r}}{2}\right) u_{j-1}^{n+2}+\frac{r}{2} u_{j-2}^{n+2} \\
& =\left(1-\frac{r}{2}-\frac{\bar{r}}{2}\right) u_{j}^{n}+\left(r+\frac{\bar{r}}{2}\right) u_{j-1}^{n} \\
& -\frac{r}{2} u_{j-2}^{n}-2 r u_{j+2}^{n+1}+(8 r+\bar{r}) u_{j+1}^{n+1} \\
& -(11 r+3 \bar{r}) u_{j}^{n+1}+(6 r+\bar{r}) u_{j-1}^{n+1} \\
& -r u_{j-2}^{n+1} \\
& \frac{r}{2} u_{j+2}^{n+2}-\left(3 r+\frac{\bar{r}}{2}\right) u_{j+1}^{n+2}+\left(1+\frac{11 r}{2}+\frac{3 \bar{r}}{2}\right) u_{j}^{n+2} \\
& -(4 r+\bar{r}) u_{j-1}^{n+2}+r u_{j-2}^{n+2} \\
& =-r u_{j+2}^{n+1}+(2 r+\bar{r}) u_{j+1}^{n+1}-(r+\bar{r}) u_{j}^{n+1} \\
& -\frac{r}{2} u_{j+2}^{n}+\left(3 r+\frac{\bar{r}}{2}\right) u_{j+1}^{n} \\
& +\left(1-\frac{11 r}{2}-\frac{3 \bar{r}}{2}\right) u_{j}^{n}+(4 r+\bar{r}) u_{j-1}^{n}-r u_{j-2}^{n}, \\
& r u_{j+2}^{n+2}-(4 r+\bar{r}) u_{j+1}^{n+2}+\left(1+\frac{11 r}{2}+\frac{3 \bar{r}}{2}\right) u_{j}^{n+2} \\
& -\left(3 r+\frac{\bar{r}}{2}\right) u_{j-1}^{n+2}+\frac{r}{2} u_{j-2}^{n+2} \\
& =-(r+\bar{r}) u_{j}^{n+1}+(2 r+\bar{r}) u_{j-1}^{n+1}-r u_{j-2}^{n+1}
\end{aligned}
$$

$$
\begin{aligned}
& -r u_{j+2}^{n}+(4 r+\bar{r}) u_{j+1}^{n}+\left(1-\frac{11 r}{2}-\frac{3 \bar{r}}{2}\right) u_{j}^{n} \\
& +\left(3 r+\frac{\bar{r}}{2}\right) u_{j-1}^{n}-\frac{r}{2} u_{j-2}^{n},
\end{aligned}
$$$$
\frac{r}{2} u_{j+2}^{n+2}-\left(2 r+\frac{\bar{r}}{2}\right) u_{j+1}^{n+2}+\left(1+\frac{5 r}{2}+\bar{r}\right) u_{j}^{n+2}
$$$$
-\left(r+\frac{\bar{r}}{2}\right) u_{j-1}^{n+2}
$$$$
=-r u_{j+2}^{n}+(4 r+\bar{r}) u_{j+1}^{n+1}
$$$$
-(7 r+2 \bar{r}) u_{j}^{n+1}+\left(6 r+\frac{\bar{r}}{2}\right) u_{j-1}^{n+1}
$$$$
-2 r u_{j-2}^{n+1}-\frac{r}{2} u_{j+2}^{n}+\left(2 r+\frac{\bar{r}}{2}\right) u_{j+1}^{n}
$$$$
+\left(1-\frac{5 r}{2}-\bar{r}\right) u_{j}^{n}+\left(r+\frac{\bar{r}}{2}\right) u_{j-1}^{n},
$$$$
-\left(r+\frac{\bar{r}}{2}\right) u_{j+1}^{n+2}+\left(1+\frac{5 r}{2}+\bar{r}\right) u_{j}^{n+2}
$$$$
-\left(2 r+\frac{\bar{r}}{2}\right) u_{j-1}^{n+2}+\frac{r}{2} u_{j-2}^{n+2}
$$$$
=-2 r u_{j+2}^{n+1}+(6 r+\bar{r}) u_{j+1}^{n+1}-(7 r+2 \bar{r}) u_{j}^{n+1}
$$$$
+(4 r+\bar{r}) u_{j-1}^{n+1}-r u_{j-2}^{n+1}+\left(r+\frac{\bar{r}}{2}\right) u_{j+1}^{n}
$$$$
+\left(1-\frac{5 r}{2}-\bar{r}\right) u_{j}^{n}+\left(2 r+\frac{\bar{r}}{2}\right) u_{j-1}^{n}-\frac{r}{2} u_{j-2}^{n} \text {, }
$$$$
\frac{r}{2} u_{j+2}^{n+2}-\left(2 r+\frac{\bar{r}}{2}\right) u_{j+1}^{n+2}+\left(1+\frac{7 r}{2}+\bar{r}\right) u_{j}^{n+2}
$$$$
-\left(3 r+\frac{\bar{r}}{2}\right) u_{j-1}^{n+2}+r u_{j-2}^{n+2}
$$$$
=-r u_{j+2}^{n+1}+(4 r+\bar{r}) u_{j+1}^{n+1}
$$$$
-(5 r+2 \bar{r}) u_{j}^{n+1}+(2 r+\bar{r}) u_{j-1}^{n+1}
$$$$
-\frac{r}{2} u_{j+2}^{n}+\left(2 r+\frac{\bar{r}}{2}\right) u_{j+1}^{n}
$$$$
+\left(1-\frac{7 r}{2}-\bar{r}\right) u_{j}^{n}+\left(3 r+\frac{\bar{r}}{2}\right) u_{j-1}^{n}
$$

$$
\begin{aligned}
& -r u_{j-2}^{n}, \\
r u_{j+2}^{n+2}- & \left(3 r+\frac{\bar{r}}{2}\right) u_{j+1}^{n+2}+\left(1+\frac{7 r}{2}+\bar{r}\right) u_{j}^{n+2} \\
& -\left(2 r+\frac{\bar{r}}{2}\right) u_{j-1}^{n+2}+\frac{r}{2} u_{j-2}^{n+2}
\end{aligned}
$$


TABLE 1: The error and the convergence rates of the ASC-N scheme.

\begin{tabular}{lcccc}
\hline$t$ & $M=201$ & $r=83.78$ & $M=401$ & $r=1327.2$ \\
& $e_{h}$ & $e_{h} / h^{2}$ & $e_{h}$ & $e_{h} / h^{2}$ \\
\hline$t=0.4$ & $1.034 \times 10^{-3}$ & 4.234 & $8.535 \times 10^{-5}$ & 1.390 \\
$t=0.5$ & $1.058 \times 10^{-3}$ & 4.331 & $8.173 \times 10^{-5}$ & 1.331 \\
$t=0.6$ & $1.039 \times 10^{-3}$ & 4.254 & $7.898 \times 10^{-5}$ & 1.286 \\
$t=0.7$ & $9.926 \times 10^{-4}$ & 4.063 & $7.573 \times 10^{-5}$ & 1.233 \\
\hline
\end{tabular}

$$
\begin{aligned}
= & (2 r+\bar{r}) u_{j+1}^{n+1}-(5 r+2 \bar{r}) u_{j}^{n+1} \\
& +(4 r+\bar{r}) u_{j-1}^{n+1}-r u_{j-2}^{n+1}-r u_{j+2}^{n} \\
& +\left(3 r+\frac{\bar{r}}{2}\right) u_{j+1}^{n}+\left(1-\frac{7 r}{2}-\bar{r}\right) u_{j}^{n} \\
& +\left(2 r+\frac{\bar{r}}{2}\right) u_{j-1}^{n}-\frac{r}{2} u_{j-2}^{n} .
\end{aligned}
$$

From the Taylor series expansion at $\left(x_{j}, t^{n+1}\right)$, we obtain the following truncation error expressions for formulae (17)(24):

$$
\begin{aligned}
& T_{\left(a a^{\prime}\right)}=\left[\frac{1}{2}\left(\frac{\tau}{h}\right)^{2} \frac{\partial^{4} u}{\partial t^{2} \partial x^{2}}\right. \\
& \left.-\frac{1}{2}\left(\frac{\tau^{2}}{h}\right) \frac{\partial^{5} u}{\partial t^{2} \partial x^{3}}+\frac{1}{2}\left(\frac{\tau}{h}\right) \frac{\partial^{3} u}{\partial t^{2} \partial x}\right]_{j}^{n+1} \\
& +O\left(\tau^{2}+h^{2}+\tau^{\alpha_{1}} h^{\alpha_{2}}\right), \\
& T_{\left(b b^{\prime}\right)}=\left[-\frac{1}{2}\left(\frac{\tau^{2}}{h}\right) \frac{\partial^{4} u}{\partial t^{2} \partial x^{2}}+\frac{1}{2}\left(\frac{\tau^{2}}{h}\right) \frac{\partial^{5} u}{\partial t^{2} \partial x^{3}}\right. \\
& \left.-\frac{1}{2}\left(\frac{\tau}{h}\right) \frac{\partial^{3} u}{\partial t^{2} \partial x}\right]_{j}^{n+1} \\
& +O\left(\tau^{2}+h^{2}+\tau^{\alpha_{1}} h^{\alpha_{2}}\right), \\
& T_{\left(a^{\prime} a\right)}=\left[-\frac{1}{2}\left(\frac{\tau}{h}\right)^{2} \frac{\partial^{4} u}{\partial t^{2} \partial x^{2}}-\frac{1}{2}\left(\frac{\tau^{2}}{h}\right) \frac{\partial^{5} u}{\partial t^{2} \partial x^{3}}\right. \\
& \left.-\frac{1}{2}\left(\frac{\tau}{h}\right) \frac{\partial^{3} u}{\partial t^{2} \partial x}\right]_{j}^{n+1} \\
& +O\left(\tau^{2}+h^{2}+\tau^{\alpha_{1}} h^{\alpha_{2}}\right), \\
& T_{\left(b^{\prime} b\right)}=\left[\frac{1}{2}\left(\frac{\tau}{h}\right)^{2} \frac{\partial^{4} u}{\partial t^{2} \partial x^{2}}+\frac{1}{2}\left(\frac{\tau^{2}}{h}\right) \frac{\partial^{5} u}{\partial t^{2} \partial x^{3}}\right. \\
& \left.+\frac{1}{2}\left(\frac{\tau}{h}\right) \frac{\partial^{3} u}{\partial t^{2} \partial x}\right]_{j}^{n+1} \\
& +O\left(\tau^{2}+h^{2}+\tau^{\alpha_{1}} h^{\alpha_{2}}\right),
\end{aligned}
$$

TABLE 2: The error and the convergence rates of the ASC-N scheme.

\begin{tabular}{lcccc}
\hline$t$ & $M=201$ & $r=837.82$ & $M=401$ & $r=1327.2$ \\
& $e_{h}$ & $e_{h} / h^{2}$ & $e_{h}$ & $e_{h} / h^{2}$ \\
\hline$t=0.05$ & $5.741 \times 10^{-4}$ & 2.350 & $1.369 \times 10^{-4}$ & 2.230 \\
$t=0.06$ & $5.947 \times 10^{-4}$ & 2.434 & $1.379 \times 10^{-4}$ & 2.248 \\
$t=0.07$ & $6.110 \times 10^{-4}$ & 2.501 & $1.379 \times 10^{-4}$ & 2.246 \\
$t=0.08$ & $6.255 \times 10^{-4}$ & 2.560 & $1.369 \times 10^{-4}$ & 2.232 \\
\hline
\end{tabular}

$$
\begin{aligned}
T_{\left(c^{\prime} c\right)}= & {\left[-\frac{1}{2}\left(\frac{\tau}{h}\right)^{2} \frac{\partial^{4} u}{\partial t^{2} \partial x^{2}}\right.} \\
& \left.+\frac{1}{2}\left(\frac{\tau^{2}}{h}\right) \frac{\partial^{5} u}{\partial t^{2} \partial x^{3}}\right]_{j}^{n+1} \\
& +O\left(\tau^{2}+h^{2}+\tau^{\alpha_{1}} h^{\alpha_{2}}\right) \\
T_{\left(d^{\prime} d\right)}= & {\left[\frac{1}{2}\left(\frac{\tau}{h}\right)^{2} \frac{\partial^{4} u}{\partial t^{2} \partial x^{2}}\right.}
\end{aligned}
$$$$
\left.-\frac{1}{2}\left(\frac{\tau^{2}}{h}\right) \frac{\partial^{5} u}{\partial t^{2} \partial x^{3}}\right]_{j}^{n+1}
$$$$
+O\left(\tau^{2}+h^{2}+\tau^{\alpha_{1}} h^{\alpha_{2}}\right)
$$$$
T_{\left(c c^{\prime}\right)}=\left[\frac{1}{2}\left(\frac{\tau}{h}\right)^{2} \frac{\partial^{4} u}{\partial t^{2} \partial x^{2}}\right.
$$$$
\left.+\frac{1}{2}\left(\frac{\tau^{2}}{h}\right) \frac{\partial^{5} u}{\partial t^{2} \partial x^{3}}\right]_{j}^{n+1}
$$$$
+O\left(\tau^{2}+h^{2}+\tau^{\alpha_{1}} h^{\alpha_{2}}\right)
$$

$$
\begin{aligned}
T_{\left(d d^{\prime}\right)}= & {\left[-\frac{1}{2}\left(\frac{\tau}{h}\right)^{2} \frac{\partial^{4} u}{\partial t^{2} \partial x^{2}}\right.} \\
& \left.-\frac{1}{2}\left(\frac{\tau^{2}}{h}\right) \frac{\partial^{5} u}{\partial t^{2} \partial x^{3}}\right]_{j}^{n+1} \\
& +O\left(\tau^{2}+h^{2}+\tau^{\alpha_{1}} h^{\alpha_{2}}\right),
\end{aligned}
$$

where $\alpha_{1}+\alpha_{1}=2$.

We briefly discuss the truncation error analysis of the ASC-N scheme. Obviously, the truncation error of the CrankNicolson scheme is $O\left(\tau^{2}+h^{2}\right)$. On the same time level, the asymmetric schemes are used symmetrically in the space direction. The signs of the terms with the parameter $h$ in (25)(32) are opposite, the effect of the terms with $h$ can be nearly canceled, and the truncation error at these boundary points is approximately $O\left(\tau+h^{2}\right)$.

4.2. The Analysis of the Stability. To prove the stability, we have to introduce the following Kellogg lemma in [19]. 


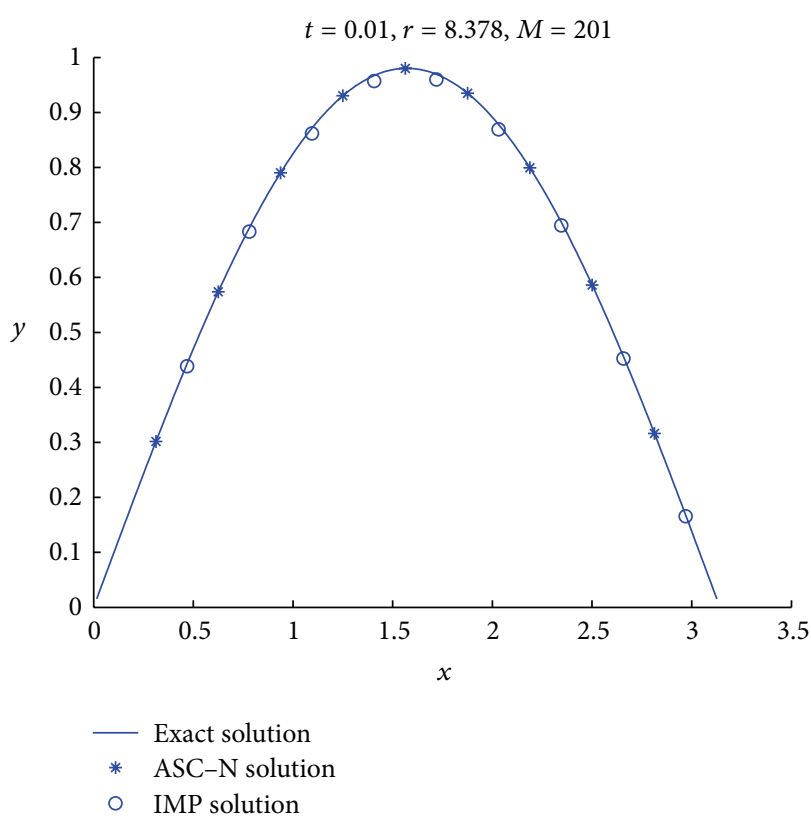

(a)

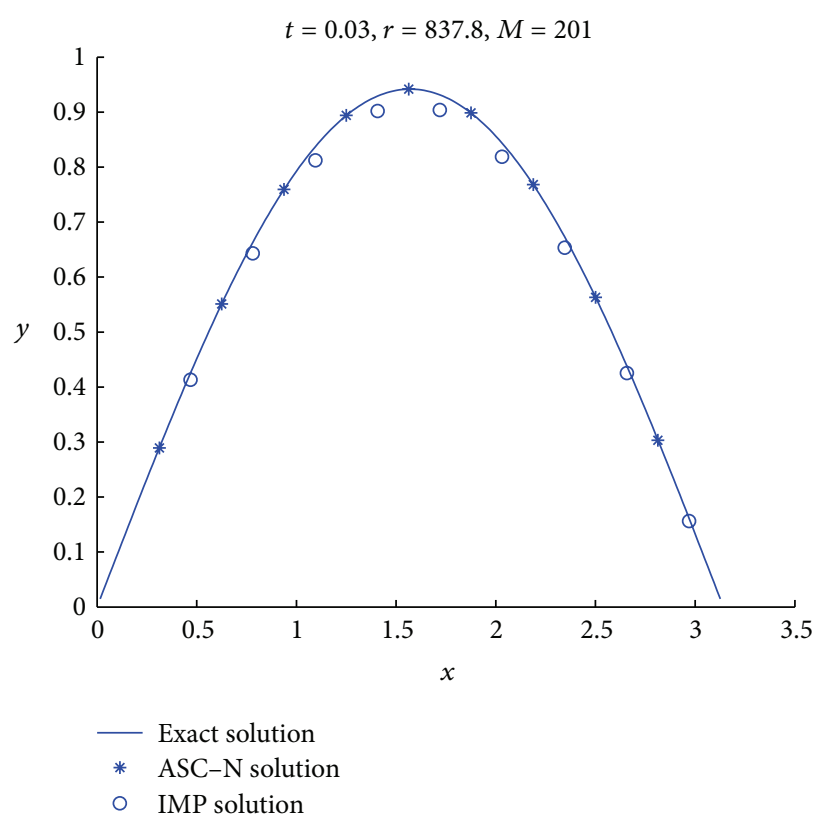

(b)

FIGURE 3: Comparison of the numerical solutions.

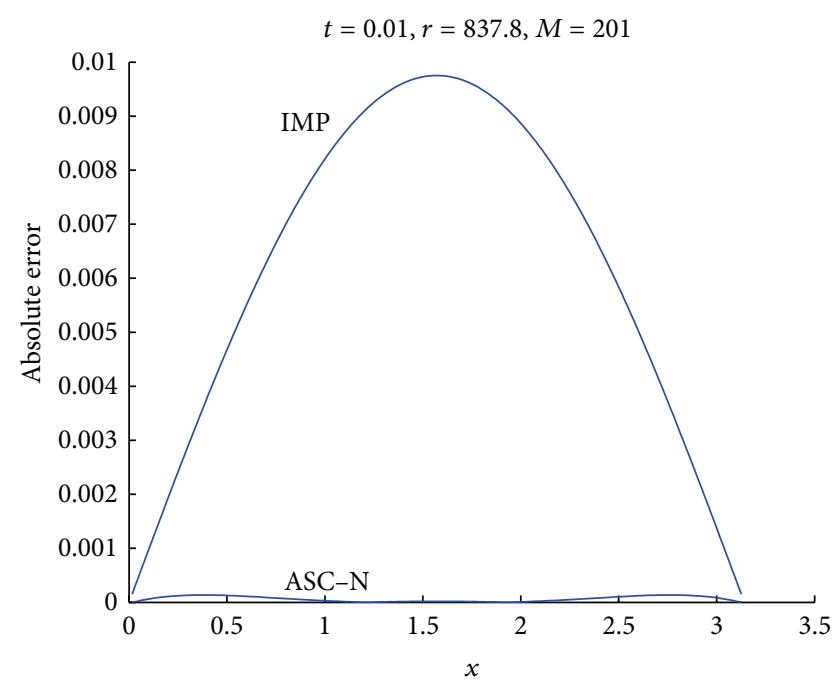

(a)

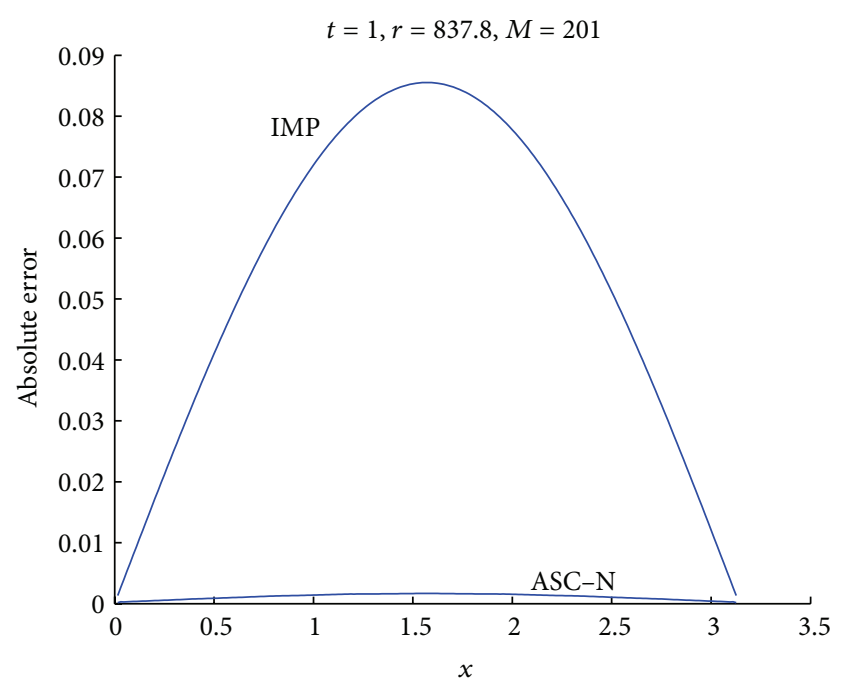

(b)

FIGURE 4: Comparison of the absolute errors.

TABLE 3: The errors of numerical solution at $M=201, r_{1}=83.78$, and $t=0.1$.

\begin{tabular}{|c|c|c|c|c|c|c|}
\hline$r$ & Errors & $j=40$ & $j=80$ & $j=100$ & $j=120$ & $j=160$ \\
\hline \multirow{2}{*}{$r=r_{1}$} & $\operatorname{Ae}\left(10^{-5}\right)$ & 3.59751 & 5.83410 & 6.18043 & 5.86366 & 3.67502 \\
\hline & $\operatorname{Re}\left(10^{-3}\right)$ & 7.50788 & 7.50789 & 7.54902 & 7.50791 & 7.50797 \\
\hline \multirow{2}{*}{$r=2 r_{1}$} & $\operatorname{Ae}\left(10^{-4}\right)$ & 1.36830 & 2.21899 & 2.35153 & 2.23023 & 1.39779 \\
\hline & $\operatorname{Re}\left(10^{-2}\right)$ & 2.85560 & 2.85561 & 2.87226 & 2.85562 & 2.85565 \\
\hline \multirow{2}{*}{$r=5 r_{1}$} & $\operatorname{Ae}\left(10^{-4}\right)$ & 4.63372 & 7.51450 & 7.98790 & 7.55253 & 4.73342 \\
\hline & $\operatorname{Re}\left(10^{-2}\right)$ & 9.67041 & 9.67040 & 9.75675 & 9.67036 & 9.67027 \\
\hline \multirow{2}{*}{$r=10 r_{1}$} & $\operatorname{Ae}\left(10^{-3}\right)$ & 1.01018 & 1.63820 & 1.74819 & 1.64647 & 1.03187 \\
\hline & $\operatorname{Re}\left(10^{-1}\right)$ & 2.10822 & 2.10820 & 2.13531 & 2.10817 & 2.10808 \\
\hline Exact & $\left(10^{-1}\right)$ & 4.79165 & 7.77062 & 8.18706 & 7.80998 & 4.89482 \\
\hline
\end{tabular}


Lemma 1. If $\rho>0$ and $\mathbf{C}+\mathbf{C}^{T}$ is nonnegative definite, then $(I+\rho \mathbf{C})^{-1}$ exists and there holds

$$
\left\|(\mathbf{I}+\rho \mathbf{C})^{-1}\right\|_{2} \leq 1 .
$$

Lemma 2. Under the conditions of Lemma 1, the following inequality holds:

$$
\left\|(\mathbf{I}-\rho \mathbf{C})(\mathbf{I}+\rho \mathbf{C})^{-1}\right\|_{2} \leq 1 .
$$

Theorem 3. For any real numbers $\bar{r}$ and $r$, the ASC-N scheme (14)-(15) is unconditionally stable.

Proof. By eliminating $\mathbf{U}^{n+1}$ from (14)-(15), we obtain $\mathbf{U}^{n+2}=$ $\mathbf{G U}^{n}$, where $\mathbf{G}$ is the growth matrix

$$
\mathbf{G}=\left(\mathbf{I}+\mathbf{G}_{2}\right)^{-1}\left(\mathbf{I}-\mathbf{G}_{1}\right)\left(\mathbf{I}+\mathbf{G}_{1}\right)^{-1}\left(\mathbf{I}-\mathbf{G}_{2}\right) .
$$

For any even number $n$, there holds

$$
\begin{aligned}
\mathbf{G}^{n}= & \left(\mathbf{I}+\mathbf{G}_{2}\right)^{-1}\left(\mathbf{I}-\mathbf{G}_{1}\right)\left(\mathbf{I}+\mathbf{G}_{1}\right)^{-1} \\
& \times\left[\left(\mathbf{I}-\mathbf{G}_{2}\right)\left(\mathbf{I}+\mathbf{G}_{2}\right)^{-1}\left(\mathbf{I}-\mathbf{G}_{1}\right)\left(\mathbf{I}+\mathbf{G}_{1}\right)^{-1}\right]^{n-1} \\
& \times\left(\mathbf{I}-\mathbf{G}_{2}\right) .
\end{aligned}
$$

Since $\mathbf{G}_{1}$ and $\mathbf{G}_{2}$ are all symmetric, $\mathbf{G}_{\mathbf{1}}+\mathbf{G}_{\mathbf{1}}{ }^{T}$ and $\mathbf{G}_{\mathbf{2}}+\mathbf{G}_{\mathbf{2}}{ }^{T}$ are nonnegative definite, so for any real number $\bar{r}$ and $r$, we can obtain the following inequality from the Kellogg lemma:

$$
\begin{aligned}
\left\|\mathbf{G}^{n}\right\|_{2} \leq & \left\|\left(\mathbf{I}+\mathbf{G}_{2}\right)^{-1}\right\|_{2} \cdot\left\|\left(\mathbf{I}-\mathbf{G}_{1}\right)\left(\mathbf{I}+\mathbf{G}_{1}\right)^{-1}\right\|_{2}^{n} \\
& \cdot\left\|\left(\mathbf{I}-\mathbf{G}_{2}\right)\left(\mathbf{I}+\mathbf{G}_{2}\right)^{-1}\right\|_{2}^{n-1} \cdot\left\|\left(\mathbf{I}-\mathbf{G}_{2}\right)\right\|_{2} .
\end{aligned}
$$

Hence

$$
\begin{aligned}
\left\|\mathbf{G}^{n}\right\|_{2} \leq\left\|\left(\mathbf{I}-\mathbf{G}_{2}\right)\right\|_{2} & \leq \sqrt{\left\|\left(\mathbf{I}-\mathbf{G}_{2}\right)\right\|_{\infty} \cdot\left\|\left(\mathbf{I}-\mathbf{G}_{2}\right)\right\|_{1}} \\
& \leq \sqrt{1+14 r+3 \bar{r}} .
\end{aligned}
$$

This shows that the ASC-N scheme is unconditionally stable.

\section{Numerical Simulations}

To illustrate the convergency, stability, and accuracy, we perform the numerical experiment for (2) using the model

$$
u_{0}(x)=\sin x, \quad L=\pi, \quad a=1, \quad \varepsilon=-1 .
$$

The exact solution of this problem is

$$
u(x, t)=e^{-2 t} \sin x .
$$

We first examine the errors and convergence rate in space for the ASC-N scheme in this paper. Let $2 l=50$; we compute the $L_{2}$-norm of the error $e_{h}=\|U-u\|_{L^{2}}$ and the convergence rate $e_{h} / h^{2}$ for different grid ratio. The results are given in Tables 1 and 2, which show that the convergence rate is approximately $O\left(h^{2}\right)$.
Next, we compare the ASC-N scheme numerical solution with the exact solution. The absolute errors (Ae) and the percentage errors $(\mathrm{Re})$ of numerical solution at $t=0.1$ are displayed in Table 3 for different $r$. From Table 3, we can see that the method given in this paper is unconditionally stable and has high accuracy.

In addition, we also compare the ASC-N solution with the implicit difference scheme (IMP) solution using the same mesh refinements. The results are displayed in Figures 3 and 4 at different time. From Figure 3 and Table 3, we can get that the ASC-N solution is nearly as good as the exact solution and is stable and reliable. From Figures 3 and 4, we also find that the ASC-N scheme has better accuracy than the IMP scheme. This is because the truncation error of the IMP scheme is $\mathrm{O}\left(\tau+h^{2}\right)$ and the truncation errors of the ASC-N scheme are also $O\left(\tau+h^{2}\right)$ at those points computed by the asymmetric scheme, but the truncation errors at those points computed by the C-N scheme are $\mathrm{O}\left(\tau^{2}+h^{2}\right)$. Consequently, we can obtain that these results agree with the theoretical analysis.

Finally, owing to the use of the asymmetric schemes, the ASC-N scheme changed the discrete problem of $M$ order into some small ( $2 l$ or $l$ order) independent problems; the parallelism of the scheme is clarity.

\section{Conclusion}

In this paper, we first constructed a group of asymmetric schemes and the C-N scheme; based on the idea of the alternating method, we gave the ASC-N scheme for the fourth-order parabolic equation. The theoretical analysis and the numerical simulations show that the ASC-N scheme constructed in this paper has high accuracy and convergence, unconditional stability, and intrinsic parallelism. The idea of this scheme is helpful for the deep study of the K-S equation.

\section{Acknowledgments}

This work is supported by the Nature Science Foundations of Tianjin of China (10JCYBJC07500 and 12JCYBJC10600) and the Foundation of Tianjin University of Technology and Education (KJ11-18).

\section{References}

[1] D. J. Evans and A. R. B. Abdullah, "Group explicit method for parabolic equations," International Journal of Computer Mathematics, vol. 14, no. 1, pp. 73-105, 1983.

[2] B. L. Zhang, "Alternating segment explicitimplicit method for the diffusion equation," Journal on Numerical Methods and Computer Applications, vol. 12, no. 4, pp. 245-251, 1991.

[3] J. Chen and B. L. Zhang, "A class of alternating block CrankNicolson method," International Journal of Computer Mathematics, vol. 45, pp. 89-112, 1991.

[4] S. Zhu, G. Yuan, and L. Shen, "Alternating group explicit method for the dispersive equation," International Journal of Computer Mathematics, vol. 75, no. 1, pp. 97-105, 2000.

[5] S. Zhu and J. Zhao, "The alternating segment explicit-implicit scheme for the dispersive equation," Applied Mathematics Letters, vol. 14, no. 6, pp. 657-662, 2001. 
[6] W. Q. Wang and S. J. Fu, "An unconditionally stable alternating segment difference scheme of eight points for the dispersive equation," International Journal for Numerical Methods in Engineering, vol. 67, no. 3, pp. 435-447, 2006.

[7] Q. J. Zhang and W. Q. Wang, "A new alternating group explicitimplicit algorithm with high accuracy for dispersive equation," Applied Mathematics and Mechanics, vol. 29, no. 9, pp. 12211230, 2008.

[8] W. Q. Wang and Q. J. Zhang, "A highly accurate alternating 6point group method for the dispersive equation," International Journal of Computer Mathematics, vol. 87, no. 7, pp. 1512-1521, 2010.

[9] W. Q. Wang, "Alternating segment Crank-Nicolson method for solving convection-diffusion equation with variable coefficient," Applied Mathematics and Mechanics, vol. 24, no. 1, pp. 32-42, 2003.

[10] W. Q. Wang, "A class of alternating group method of Burgers' equation," Applied Mathematics and Mechanics, vol. 25, no. 2, pp. 236-244, 2004.

[11] F. L. Qu and W. Q. Wang, "Alternating segment explicit-implicit scheme for nonlinear third-order KdV equation," Applied Mathematics and Mechanics, vol. 28, no. 7, pp. 973-980, 2007.

[12] G. Y. Guo and B. Liu, "Unconditional stability of alternating difference schemes with intrinsic parallelism for the fourthorder parabolic equation," Applied Mathematics and Computation, vol. 219, no. 14, pp. 7319-7328, 2013.

[13] G. Y. Guo, Y. S. Zhai, and B. Liu, "The alternating segment explicit-implicit scheme for the fourth-order parabolic equation," Journal of Information and Computational Science, vol. 10, no. 10, pp. 3125-3133, 2013.

[14] Y. Kuramoto and T. Tsuzuki, "On the formation of dissipative structures in reaction diffusion systems," Progress of Theoretical and Experimental Physics, vol. 54, pp. 687-699, 1975.

[15] G. I. Sivashinsky, "Nonlinear analysis of hydrodynamic instability in laminar flames. I: derivation of basic equations," Acta Astronautica, vol. 4, no. 11-12, pp. 1177-1206, 1977.

[16] R. C. Mittal and G. Arora, "Quintic B-spline collocation method for numerical solution of the Kuramoto-Sivashinsky equation," Communications in Nonlinear Science and Numerical Simulation, vol. 15, no. 10, pp. 2798-2808, 2010.

[17] B. Soltanalizadeh and M. Zarebnia, "Numerical analysis of the linear and nonlinear Kuramoto-Sivashinsky equation by using differential transformation method," International Journal of Applied Mathematics and Mechanics, vol. 7, pp. 63-72, 2011.

[18] M. Lakestania and M. Dehghanb, "Numerical solutions of the generalized Kuramoto-Sivashinsky equation using B-spline functions," Applied Mathematical Modelling, vol. 36, no. 2, pp. 605-617, 2012.

[19] R. B. Kellogg, "An alternating direction method for operator equations," SIAM, vol. 12, no. 4, pp. 848-854, 1964. 


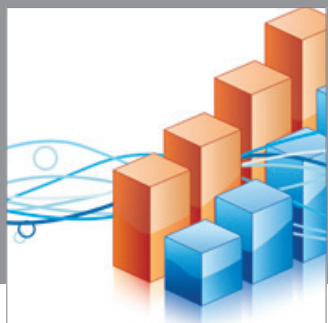

Advances in

Operations Research

mansans

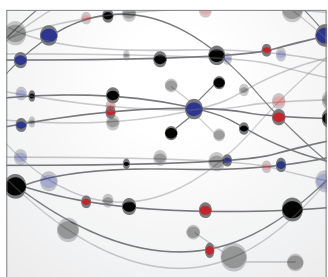

The Scientific World Journal
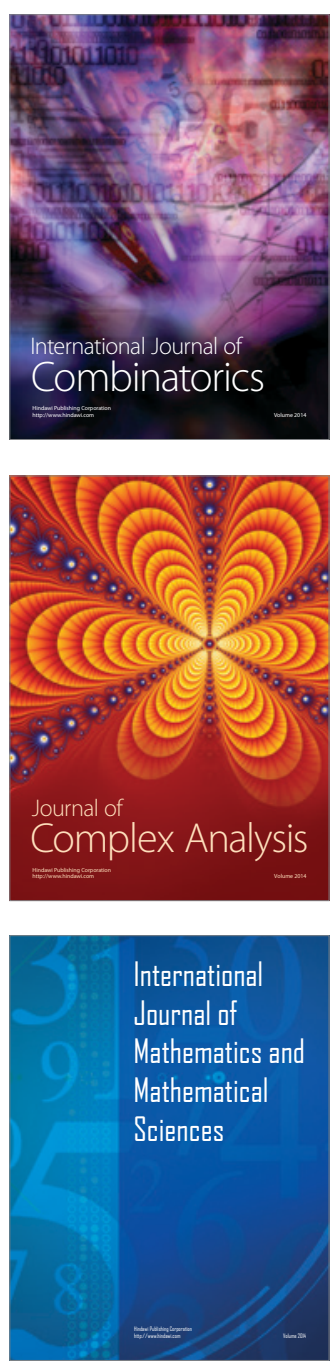
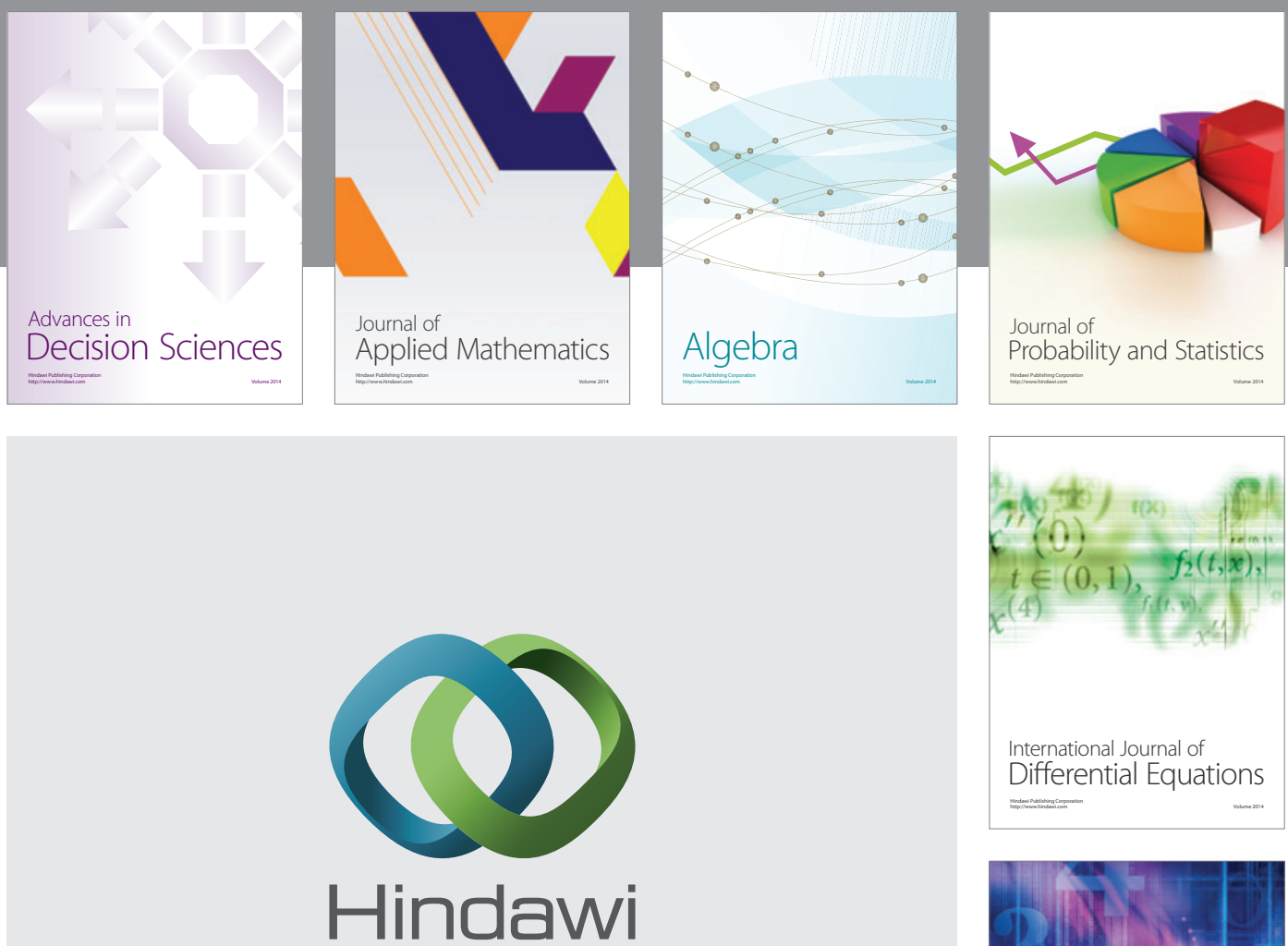

Submit your manuscripts at http://www.hindawi.com
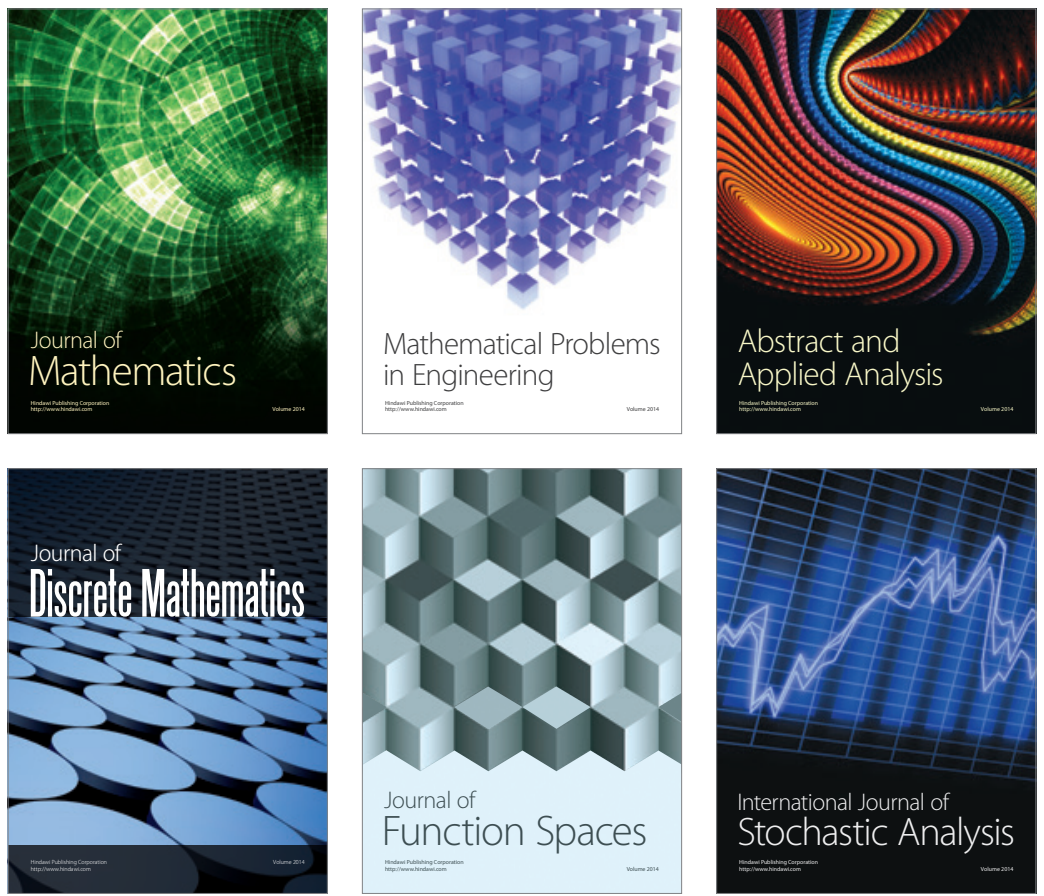

Journal of

Function Spaces

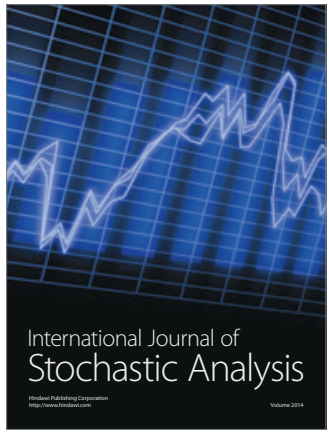

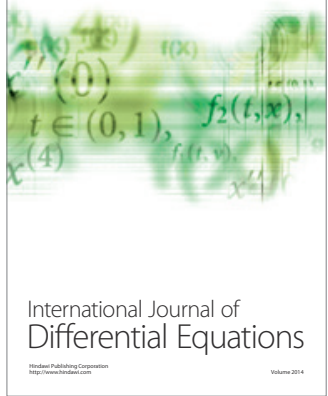
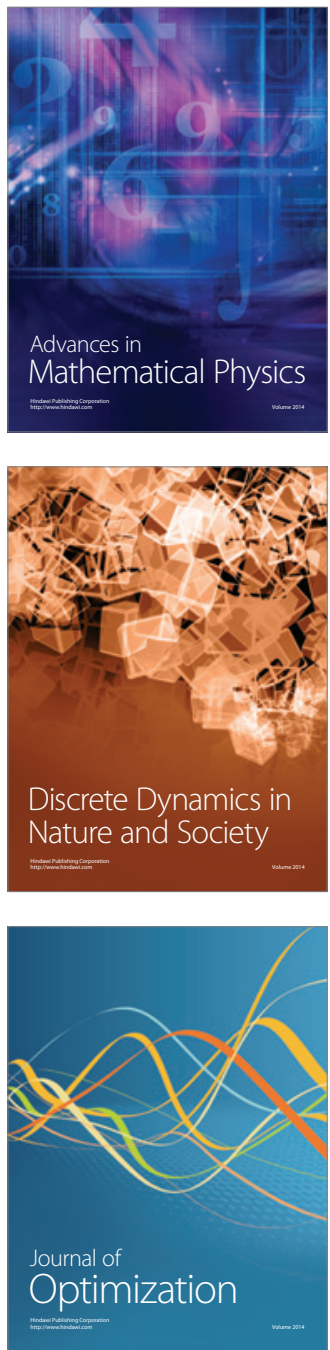